\title{
Content Identifier
}

National Cancer Institute

\section{Source}

National Cancer Institute. Content Identifier. NCI Thesaurus. Code C99023.

The unique identifier for a piece of content such as a document, image, or other media in a specified context. 